\title{
How to be a realist about Minkowski spacetime without believing in magical explanations
}

\author{
(Cómo ser realista sobre el espacio-tiempo de Minkowski sin creer en explicaciones mágicas)
}

\author{
Adán Sus*
}

\begin{abstract}
The question about the relation between spacetime structure and the symmetries of laws has received renewed attention in a recent discussion about the status of Minkowski spacetime in Special Relativity. In that context we find two extreme positions (either spacetime explains symmetries of laws or vice-versa) and a general assumption about the debate being mainly about explanation. The aim of this paper is twofold: first, to argue that the ontological dimension of the debate cannot be ignored; second, to claim that taking ontology into account involves considering a third perspective on the relation between spacetime and symmetries of laws; one in which both terms would be somehow derived from common assumptions on the formulation of a given physical theory.
\end{abstract}

KEYWORDS: Special Relativity; Minkowski spacetime; Dynamical approach; Explanation; Spacetime Symmetries; Dynamical Symmetries.

RESUMEN: La pregunta sobre la relación entre las simetrías epaciotemporales y las simetrias de las leyes ha recibido atención renovada en la reciente discusión sobre el estatus de la métrica de Minkowsi en la Teoría de la Relatividad Especial. En ese contexto, encontramos dos posiciones extremas (o bien las simetrías espaciotemporales explican las simetrías de las leyes o viceversa) y el supuesto general de que el debate tiene que ver primariamente con la cuestión de la prioridad explicativa. El objetivo de este artículo es doble: primero, argumentar que la dimensión ontológica del debate no puede ser ignorada; segundo, defender que atender a esta dimensión implica considerar una tercera perspectiva sobre la relación entre espaciotiempo y simetrías, en la cual ambos términos se derivarian de los presupuestos implicados en la formulación de cierta teoría física.

PALABRAS CLAVE: Relatividad Especial; Espaciotiempo de Minkowski; Perspectiva Dinámica; Explicación; Simetrías espaciotemporales; Simetrías Dinámicas.

* Correspondence to: Adán Sus. Departamento de Filosofía, Universidad de Valladolid, Plaza del Campus, s/n (47011 Valladolid-España) adansus@fyl.uva.es - https://orcid.org/0000-0002-2097-1192

How to cite: Sus, Adán (2020). "How to be a realist about Minkowski spacetime without believing in magical explanations»; Theoria. An International Journal for Theory, History and Foundations of Science, 35(2), 175-195. (https://doi.org/10.1387/theoria.21065).

Received: 06 August, 2019; Final version: 20 February, 2020

ISSN 0495-4548 - elSSN 2171-679X / (C) 2020 UPV/EHU

This article is distributed under the terms of the

Creative Commons Atribution 4.0 Internacional License 


\section{Introduction}

The philosophical discussion about the nature of space and time in physical theories has been historically situated on a terrain defined by two extreme positions, usually characterised as substantivalism and relationalism. These labels define two different attitudes towards the ontology of space and time that can be simply characterised as follows: either space and time are entities whose existence is independent from the other entities assumed to exist in the world or they are somehow derived or emergent from some properties of these other existents. This discussion has had a convoluted history and has taken different shapes in the contexts of different theories. The aim of this paper is twofold: explore the middle territory between these two extreme positions to vindicate the availability of a perspective that is not a temperated version, or a mixture, of any of the other two; and do so by confronting one of the recent incarnations that the debate has taken in the context of relativity theory.

In the context of Special Relativity (SR) we find a recent related discussion about the status of the spacetime metric. The standard (realist) received view, a.k.a. geometrical approach (GA), here represented by Janssen (Balashov et al., 2002; Janssen, 2002, 2009), would maintain that spacetime is somehow responsible for the default motion of bodies and for special relativistic phenomena like length contraction and time dilation. On the other side, the constructivist/dynamical approach (DA), put forward by Harvey Brown and Oliver Pooley (Brown, 2005; Brown et al., 2006), challenges this view by pointing that typical special relativistic phenomena can be said to get a dynamical explanation in the context of SR. Recent discussions about this debate can be found in (Acuña, 2016; Dorato et al., 2010; Felline, 2011, 2015; Frisch, 2011;Myrvold, 2017; Norton, 2008; Sus, 2019).

What is exactly the discussion about? The initial terminology seems to suggest that the main problem is ontological: what is the nature of spacetime and how to understand its relation to certain effects and objects. And, no doubt, this is one notorious dimension of the discussion. Nonetheless, the main contenders explicitly say that the dispute is also (or, sometimes, mainly) about explanation: whether some features of spacetime can be said to explain special relativistic effects or, to the contrary, they are explained, in the theory, by some features of the (matter) laws. The dispute, then, is said to be about the arrow of explanation: either it goes from the symmetries of Minkowski metric to Poincaré invariance of laws or vice versa. One can also present the debate in terms of the basic assumptions that the theory makes: the standard view would say that the spacetime structure (Minkowski in SR) with its symmetries is a brute fact in the theory, the dynamical approach would pose as a primitive fact the Poincaré invariance of all matter laws. The first task of this paper will be to evaluate the relative weight of the ontological and explanatory dimensions in the present debate.

In the first part of the paper I will confront the discussion about the arrow of explanation in the terms posed by Janssen. His main point is that the arrow goes from the symmetries of spacetime metric to the invariance of laws, although his justification for the Minkowski metric being the explanans can seem somehow enigmatic: in SR, Minkowski metric explains by re-drawing the line between kinematics and dynamics and separating those effects that do not need dynamical explanation from the dynamical effects. Is this really an explanation? I will defend that this account is not sufficient to support the 
claim about the arrow of explanation going from the metric to the symmetries of laws. The model of explanation implicit in Janssen's defense of the explanatory import of Minkowski metric seems to be provided by something like the notion of structural explanation. Even if correct, I will argue that its ontological neutrality does not allow us to distinguish such an account from the so-called truncated explanation contemplated in the dynamical approach; basically, the claim that spacetime has the structure it has, Minkowskian in SR, because the dynamical laws are Lorentz invariant. The difference then, from this perspective, seems merely terminological.

I will also look at the ontological dimension independently. Norton (2008) forcefully argues that the constructivist project fails and he does so by putting the focus on the ontological question. His main claim is that constructivists (or defenders of the DA), to have a real alternative proposal to the realist one, should be able to infer the full spacetime structure from the matter theories without presuming any of the properties that, according to the realist, are properties of the spacetime structure. Norton shows how the constructivist project needs to inject spatiotemporal assumptions in order to get off the ground. Even agreeing with Norton, I think that his realists implications are too strong. I will try to show that an analysis akin to Norton's does not give too much comfort to the (naïve) realist.

The first part of the paper ends claiming that neither of the two positions seems to offer a complete satisfactory answer to the question about the relation between spacetime symmetries and the symmetries of laws. On the side of the geometrical approach, the ontologically burdened naïve realist does not provide any account of such relation while the modest, ontologically neutral, position - defended by Janssen - points to a structural explanation of the effects but is too weak to sustain any claim about the arrow of explanation. For the dynamical approach the problem comes from stressing the need for a dynamical explanation while taking the symmetries of laws, which seem to sustain the explanatory claims, as brute facts. ${ }^{1}$ This situation paves the way to explore a middle ground proposal: one able to fill the explanatory gap left by the realist and to provide an account of the common origin of the symmetries of laws assumed to be primitive by the constructivist. The second part of the paper is dedicated to this.

I will argue that the previous discussion suggests looking for a constructivist view that supports some kind of structural explanation but that regards the Minkowski metric, as well as the symmetries of laws, as both deriving from common assumptions. The sketch of one such a proposal is the following: it follows the idea of spacetime structure being composed by a set of events on which different geometrical structures are imposed. But now the project consists on tracking down the origin and justification of such structures; and the leading idea is that this is provided by some notion of physical experience. Thus the scheme traces the justification of the spacetime structures to the notion of physical experience which is ingrained in the different definitions of physical events. Furthermore, these conditions can be seen, also, as conditions for the formulation of physical laws (which provide relations between events). In this way, we are able to present a description of the common origin of the symmetries of laws and the spacetime symmetries. Although this kind of

1 As Acuña (2016) stresses, the full version dynamical approach would take the explanation of relativistic effects as provided in the context of a final quantum theory of matter. In that situation, the brute fact would be having such fundamental laws with their invariance properties. 
perspective is far from being new, I believe that it has not been sufficiently considered in recent debates about the foundations of spacetime. ${ }^{2}$

\section{Explaining without explanation. The kinematics/dynamics division}

Janssen (2009) can be seen as providing a defense of the standard view of the role of spacetime structure in SR. His position can be summarised by saying that although Minkowski metric explains the symmetries of laws, and such symmetries are responsible for the derivation of the special relativistic effects, this does not involve having a strong ontological commitment with respect to spacetime. The explanatory scheme that sustains such claim is what Janssen calls common origin inference (COI), a sub-species of inference to the best explanation. In our case, the inference would go from the invariance properties of matter laws to the symmetries of spacetime (the common origin). As Janssen acknowledges, quoting Brown and Pooley, from this initial take it is not clear in what sense bringing about the Minkowski metric provides an explanation of the invariance of laws; not, unless one gives a story of what the relation between symmetries of spacetime and the invariance of laws is. In other examples of inference to the best explanation, one might infer to a common origin, but one still needs a good explanatory story in place.

Janssen thinks that his position on the explanatory role of Minkowski metric does not involve any commitment with respect to the ontology of the metric. This neutrality is in part attributable to the fact that the explanation that Janssen has in mind is not a causal one. If this were the case, it would be easier to accuse him of reification of the metric and easier for the critics to link the destiny of his position to that of substantivalism. Even if it is true that taking distance from the account of causal explanation can protect you from the direct attacks of the relationalists, this does not alleviate the need of providing a detailed account of how the explanation is supposed to work. Moreover, only then we will be able to judge to what extent the proposal is free from ontological commitments.

What Janssen has in mind as for the model of explanation is what has been named structural or geometrical explanation.

[...] the universality of the Lorentz-FitzGerald contraction in special relativity is no more miraculous than that right-angled triangles cut out of flat sheets of paper, plastic, and card board all satisfy the Pythagorean theorem of Euclidean geometry. (Janssen, 2009, p. 49)

Nonetheless, it is not clear whether Janssen wants to take the geometrical analogy too far or too deep. In response to the challenge of showing how the Minkowski metric is supposed to explain or why it should provide any gain with respect to the explanation by the symme-

2 There is a long tradition of attempts that try to derive relativistic spacetime starting from certain principles or assumptions that have to do with causality or connectivity between events (See Section 5 for references). My claim is that such approaches are close to the perspective introduced here as a third way between the realist and the constructivist camps. To appeal to the notion of possible experience can be seen as introducing an unfortunate subjective dimension in the discussion. I hope that the use I make of it dissipates these concerns. 
tries of the laws, the answer seems to be that in SR a full set of phenomena is explained by declaring them kinematical, default behaviour or, in other words, as not in need of a dynamical explanation. So the set of kinematical phenomena contains the default behaviour of bodies and this happens to be codified by Minkowski geometry. Nonetheless, it seems necessary to show what the explicit connection between Minkowski structure and the kinematical/dynamical divide is.

We might express the situation in the following way. Janssen's account, taken literally, does not seem to capture the ontic dimension of explanation at all; although this might not be bad in itself, such dimension seems essential for the story about the arrow of explanation. The reason is that even if it was right that some features of the laws, encoded by the Minkowski metric, are explanatorily relevant, it is not clear in what sense this would support the idea of having an arrow of explanation going from spacetime to the laws. According to Janssen's version of the geometrical approach, some features of the matter laws (their Poincaré invariance) do directly the explanatory job and, somehow, Minkowski spacetime further explains these properties of the laws. But then the task to do is to explore what exactly, if there is one, the explanatory connection between spacetime geometry and the symmetries of laws is.

\section{How can Minkowski spacetime explain structurally?}

It seems clear that the main hope for providing an account in which special relativistic effects are explained by means of Minkowski metric rests on fitting this case in a non-causal scheme. There is not a unitary account for non-causal explanations in the literature, even if many of them seem to fall under some type of mathematical or formal explanations. Nevertheless, even restricting our attention to this class, we find important divergencies with respect to what would be involved in such explanations.

I will use the general label formal explanation to refer to any account of scientific explanation that is non-causal (the relation between explanans and explanandum is not a causal one) and presumes that the explanation of the given phenomenon is due to some formal or mathematical feature. Most of the accounts based on this idea of formal explanation recognise that the key question that they must answer has to do with the relationship between the explanatory formal features and the structure of the explained phenomena. Let us take as a significative example Bokulich's (2011) model based structural explanation, and see how it can be made to work in the context of Special Relativity (Dorato et al., 2010; Felline, 2011, 2015).

Bokulich makes the conditions for a model to be explanatory explicit. She contemplates the possibility of having different types of explanatory models (some mechanistic or causal) including some explaining structurally, and intends to provide a general framework that can accommodate all of them. The basic assumption to be able to take a model as explanatory is "that the counterfactual structure of the model is isomorphic (in the relevant respects) to the counterfactual structure of the phenomenon" (Bokulich, 2011, p. 23). So the idea is that for a model to be explanatory it must capture those aspects on which the phenomenon depends counterfactually; that is, such that if changed would change relevant aspects of the phenomenon. This constitutes a structural explanation in case the counterfactual dependence is between the structural or formal features of the phenomenon and 
some of those same features of the theories employed to model the phenomenon. So once we assume that the structure of the phenomenon is isomorphic to some relevant structural features of the model, we do have the structural explanation. And presumably this correspondence is part of what determines that the model is a model of the phenomenon. Then it is part of the explanation that the model represents the phenomena. The question of interest for us is whether this is enough to understand the explanatory role that Minkowski metric seems to play. Let us see how to apply this to Minkowski's spacetime. ${ }^{3}$

Recall that in the case of Minkowski metric what is at stake is whether this structure can be said to explain special relativistic effects. In Janssen's two steps strategy, insofar as such effects are a direct consequence of the symmetries of the laws, we should be happy if we were able to explain such symmetries starting from Minkowski metric; taking for granted that the symmetries of laws must mimic those of the metric does not provide an explanation of them in terms of the metric. So one must be more subtle if one wants to search for a structural explanation in this case. What seems clear, so far, is that any account of the special relativistic effects has some formal features in common: it assumes that the relations between events must satisfy the rules of Minkowskian geometry. And, arguably, this is what constitutes the counterfactual structure of the phenomenon mentioned above, which is what would make Minkowsky metric part of the explanans, according to Bokulich.

This is the core of conceiving explanations of effects by Minkowski spacetime as structural ones in SR. But it must be noted that if this is how the Minkowski metric explains, it is very far from the supposedly realist spirit that is opposed to the constructivist account. In fact, it does not seem to go beyond the epistemic notion of explanation ${ }^{4}$ and, without further elements, to say that it is Minkowski structure what does the explanatory work instead of the symmetries of the laws might be seen as a terminological subtlety (unless one understands such a structure as being a property of events or laws, as we will consider below).

Let me insist on this last point. Attending to the previous discussion, it is not difficult to see why it seems natural to regard the explanation of special relativistic effects as a genuine geometrical, therefore formal, explanation; as Janssen says, the features that we take as explananda are of the same type as the ones that we mention when we want to explain that the sum of the angles of a triangle is $180^{\circ}$. So far, the thing does not seem very controversial. Nonetheless, there is a detail that should not be forgotten: such an explanation either presupposes that physical events satisfy the rules of a given geometry (be it either Euclidean or Minkowskian) or it derives the effects from the invariance properties of laws. More precisely, what this type of explanation does is to show that some effects, that are consequence of certain properties of laws, can be described in geometrical terms, or that they are essentially geometrical. Perhaps this is all there is about the explanation of the effects in SR; they are explained by being declared geometrical. But this is neither better nor worse than saying that in SR the effects are explained because, if the theory is right, it imposes the formal condition of Poincaré invariance to any physical law. I suspect, nonetheless, that this is much less than what is usually implied when, in the context of SR, it is argued that the arrow of

See, for instance (Dorato et al., 2010; Felline, 2015).

4 One might think that this should not come as a surprise as we are using a model based account of explanation and, as such, the notion of explanation involved can only be epistemic. I am not saying that this should be taken as a defect, but that it is not enough to account for the ontological dimension of the problem. 
explanation goes from Minkowski metric to the symmetries of laws. It is at least questionable that opposition to the dynamical approach can be defended with such weak reading of the structural explanation, even if it is true that it is claimed (Janssen, 2009; Felline, 2011) that the position is ontologically neutral. In any case, independently of which ontological agenda is behind the defenders of the viability of this type of explanation for relativistic effects, what seems unquestionable is that this does not support the idea of spacetime symmetries explaining that the symmetries of laws are such and such, rather than the claim of some formal features of laws explaining certain effects.

Therefore, the model of structural explanation in itself applied to the case of SR does not have any strong import on the question of the arrow of explanation and, furthermore, there is no reason to extract from this the ontological moral that naïve realists extract; namely, that Minkowski spacetime, understood as something different from the mentioned formal features of laws, is part of the explanation. Recent discussions of the dynamical approach (Read, preprint; Brown et al.) seem to agree on this verdict about the geometrical approach. In (Read, preprint) it is argued that GA might take a defensible version (there named qualified geometrical approach) if it is assumed from the beginning that all matter laws are Poincaré invariant (this fact is named in (Read et al., 2018) and (Brown et al., forthcoming; Read, preprint) as the first miracle of relativity) and Minkowski metric is then used as part of the explanations of the relativistic effects. This version of GA, then, would combine giving up to explain the coincidence of the invariance properties of matter laws (one of the apparent original motivations for the geometrical approach) with defending the explanatory priority of the Minkowski metric. It is not clear to me what the appeal of such a position would consist of. As it is claimed in (Read, preprint) such an approach would be only distinguishable from the dynamical approach, in the context of SR, in the claim of ontological autonomy of the metric without seeking to explain from it the coincidence of the invariance properties of laws; introducing, hence, two unexplained coincidences: the symmetries of all matter laws (miracle 1) and that they are the same as the symmetries of the metric (miracle 2). Even if this position is viable, it is hard to see it as well motivated.

Nonetheless, it is still true, as Janssen stresses, that as one can argue that effects are derivable from some formal features of the laws, with independence of their specific content, the defenders of the dynamical approach need to refine their claim of this constituting a dynamical explanation. In the second part of the paper, I will propose what I think is a better ontological embodying of such structural explanations in SR; one that does not fit so comfortably in the standard realist mould.

\section{From explanation to ontology}

We have seen how the debate about the relation between Minkowski spacetime and laws is presented in terms of explanatory priority and how it is assumed that one can deal with it leaving ontology out. Nevertheless, considering that the status of spacetime is under the focus, it seems difficult to ignore the ontological dimension. John Norton (2008) suggests that the discussion between defenders of the geometrical and the dynamical approaches is primarily about ontology, or at least that the ontological import is unavoidable, and recommends to leave out the difficult issues about explanation. I partly agree with this: keeping 
ontology out of the equation empties the discussion to the risk of rendering it merely terminological. One must recover ontology then, but the key issue is how to reintroduce it in the dispute. I will try to show that doing it right illuminates the original controversy.

First, it must be stressed that leaving out the question of ontology makes considerably difficult to say what the difference between constructivists (using Norton's way of referring to what, according to him, would be a coherent version of DA) and their critics is about. $^{5}$ Right, one can say that the difference is about the direction of the arrow of explanation - whether it goes from the symmetries of the metric to the symmetries of the laws or vice versa - but we have seen that trying to give content to the realist explanatory claim, and doing it in terms of a formal explanation scheme (which seems the only reasonable alternative), brings the position suspiciously close to the constructivist one. The quid for explaining the relativistic effects by the metric is rooted in the claim to the effect that every model of the theory shares the same chronogeometry (Minkowski), i.e. the claim stating the existence of an isomorphism between formal structure of the models and the geometrical structure. Obviously, one can express this fact by stating that all the models of the theory are solutions of certain equations that share a formal feature: Poincaré invariance. And unless one introduces some extra element to provide more weight to one or the other side of the explanation - the geometry or the invariance properties of the laws - it does not seem that we have a proper substantive difference. ${ }^{6}$ Explanatory priority without ontological commitments seems empty, but introducing ontological preferences can at this stage seem arbitrary. ${ }^{7}$

One place in the literature where the ontological dimension of the discussion is placed upfront is Norton's paper (2008). There, the core of the constructivist program is presented by opposition to the realist conception of Minkowski spacetime; and the failure of such a program is sketched as the impossibility of constructing the special relativistic spacetime from matter fields without introducing spacetime assumptions from the start. On top of this, Norton provides a clear statement of which spacetime presuppositions would enter in the constructivist derivation of spacetime from the matter laws.

The starting point for this kind of analysis is a clear opposition between the realist perspective (assumed to be less problematic) and a strong constructivist program. The perspective then is ontological from the start as it presents the dispute as consisting on a divergence about what the basic entities of the world are. The rationale for presenting things this way is, according to Norton, that one avoids the undefined terrain of the never ending discussions about the right notion of scientific explanation and, then, one can formulate what a definite constructivist position would be by opposition to the realist one. And if

5 A similar criticism about the alleged ontological neutrality of the debate can be found in Acuña (2016).

6 At least not if we consider the truncated version of the geometrical approach and restrict attention to SR. Whether the full version would be substantivally different is something that can only be addressed after letting in the question about ontology. This possibility is explored in (Read, 2019); Sus (Preprint).

7 I am not denying that in many contexts showing that a given class of phenomena share a common feature can be explanatory: unifying somehow different situations is one of the rewards provided by scientific explanations. What I am arguing against is the idea of this providing an account of explanations that is substantively different to the one offered by the dynamical approach. 
one reminds our disappointing conclusion from the last section - that from the purely explanatory perspective the two positions either collapse or rest on non-explicit ontological assumptions - one might welcome this new approach.

Let us see what Norton finds as spatiotemporal assumptions in the constructivist program and later we can attempt a different justification for them. The strong constructivist project is, then, to build Minkowski spacetime purely from matter laws. Norton's strategy is to make explicit how such a project requires spatiotemporal assumptions. According to him, the constructivist must start with a set of matter theories, that are Lorentz invariant, and try to identify elements in them that would correspond to clocks and rods: this would allow to produce the quantity that can be identified with Minkowski's spacetime interval, and from it the full Minkowski metric would follow. Leaving aside the question of whether the theory allows structures that can be identified with stable clocks, what Norton notes is that in one such a construction there is already quite a lot of spacetime presupposed. First, he asks, how can we know that the parameters of the different matter theories identify the same physical events; or, in other words, that we have a unique set of parameters that can be used for all the matter theories? This is a natural thing to accept if one assumes that every matter theory inhabits the same spacetime (if the parameters are given spatiotemporal meaning from the start). But, as Norton points out, this is question begging for the strong constructivist. Let us remark that this assumption, from the formal point of view, can be referred as the necessity of introducing a topological manifold of events with coordinate charts.

Norton also argues, I think that less convincingly, that to make full sense of their program strong constructivists should either think of space and time intervals as properties of a spacetime entity (which is clearly not consistent with their view) or be committed to a very strong form of operationalism in which quantities have no values unless they are actually measured. The argument combines the intuitions behind the COI arguments discussed above (that any matter theory measures the same intervals should be taken as evidence in support for the existence of a common origin) and the idea that the constructivist could not use spacetime notions for empty regions of spacetime (there would be no time interval between two events unless there is an actual clock traveling between them).

We can take this discussion as a way of identifying some challenges that an alternative to the account of special relativistic effects in terms of the Minkowski metric must face. It must justify the necessity of introducing something equivalent to the spacetime manifold of events and must provide an account of space and time notions in a way that does not present them as the result of measuring a previously given spacetime entity to which matter fields adapt. In what follows, I will show how to meet these requirements without importing the problems of the naïve realist when trying to provide an account of the explanatory power of Minkowski metric.

\section{Explaining spacetime?}

This is the situation so far. The dynamical approach (or constructivist program) would defend that, in SR, relativistic effects are explained by the condition that all matter laws must be (locally) Poincaré invariant. The problem for such an approach is that, taken as a brute 
fact, such a restriction on laws seems an unacceptable cosmic coincidence. ${ }^{8}$ On the other side of the dispute, the defense of the explanatory power of Minkowski spacetime either takes a full realist flavour and is in need of providing a story of the connection between spacetime and symmetries of laws, or it tries to be ontologically neutral and advocates for a formal notion of explanation. I think that the latter is the most promising strategy but I disagree with its defenders' aim of leaving ontology out of the discussion, if what they want is to distinguish themselves from the dynamical approach. I have argued that if one does so, the notion of explanation involved in the claim is so thin that its difference with what is defended in the dynamical approach seems merely terminological: no substantive distinction regarding the arrow of explanation is found. What the dynamical approach needs, then, is an account of the coincidence of the symmetry of every law (the common origin); on the other hand, what the realist position needs is filling in its epistemic account of the explanatory relevance of Minkowski metric. One thought that seems natural is that both needs point in the same direction: what makes geometry Minkowskian and what makes the matter laws Poincaré invariant. Is there a common origin for these two facts? I want to argue that this is the case and show how looking for such an origin involves developing a truly constructive strategy in a different ontological setting. ${ }^{9}$ What I present in the rest of this paper is a description of a program to attempt that, not a full-fledged proposal.

Let me assume what I believe is a natural ontological framework for physical theories. For our purposes, we will take a physical theory as providing a systematic description of the relation between physical events (occurrences). It is obvious that physical theories exhibit varied ontologies, but we can assume that the idealised notion of event is sufficiently general to be considered as the basic block for a great number of them. It is common in modern presentations of spacetime theories to take the notion of event as a fundamental conceptual starting point. Given that, the relevant question is how to charactarise such events and how much ontological import give to them. Sometimes events are seen as mere conceptual tools, others, the notion has a stronger ontological burden (Dorato, 2015). A realist conception of spacetime can give the notion of event a central role too: there an event is understood as a generalisation of the concept of geometrical point of a substantival space. This usually comes together with a substantivalist conception of spacetime: the idea of events being spacetime points is translated literally into ontological terms, understanding spacetime as an entity composed by events. But it is not compulsory, not even advisable, to interpret the notion of event in these terms.

So one can think of the notion of spacetime event as a trademark of a substantivalist perspective. But, is there an alternative way of reading the role played by the notion of spacetime event? After all, initially an event is nothing else than a possible physical occurrence. From this initial characterisation it should be clear that in the notion of event the spatio-

8 As mentioned above, such a coincidence is meaningfully labeled in recent developments of the approach (Read et al., 2018; Read, 2019, preprint) as the first miracle of relativity.

9 It is true, as Acuña (2016) notes, that the problem of giving account of the symmetries of matter laws being the same is one for the truncated version of the constructivist approach, tenable because there is not yet a full quantum theory of matter, but would not be so for a full version in which Lorentz invariance might be taken as derived from such a fundamental theory. Nonetheless, either in the full version Lorentz invariance is a brute fact, in which case it is not that different from the situation in the truncated version, or it is derived and one can conjecture the type of explanation that would be provided. 
temporal aspect of reality is linked to the modal dimension of laws. That events are possible physical occurrences means that they are occurrences allowed by the physical theories in question, and somehow encoded in their equations of motion; it is only in conjunction with this that they can be taken as components of spacetime. Arguably, this is what is ultimately alluded to in statements that present spacetime as the set of all possible events. It is possible to understand this in substantivalist terms, by somehow separating the spatiotemporal dimension from the laws, but in the context of our discussion one must keep in mind that such structure is nothing more than an abstraction from the collection of physical events, understood as possible occurrences in the context of some physical theory. This provides a conceptual framework to understand the relations between spacetime structure and physical laws.

This slight switch of perspective - from engaging directly with the question about the nature of spacetime to regarding this issue as deriving from a reflection on the conditions that allows the formulation of the different physical theories - has its origins in Kant's analysis of experience and a long tradition that arrives to our days; specially relevant are the discussions of Michael Friedmann and Robert DiSalle for the translation of Kantian ideas to the relativistic context. Here, I do not intend to enter in the discussion of these different proposals or to track the connections (which are nevertheless indisputable) with my own. ${ }^{10}$ My only intention here is to sketch a general framework which I believe to be specially illuminating for the discussion of the relation between spacetime symmetries and symmetries of laws. It can be called, in general, a trascendental approach. ${ }^{11,12}$

The starting point, then, is the idea of investigating the nature and structure of spacetime understood as a set of features of the collection of possible physical events. This provides an alternative ontological perspective to taking spacetime structure as primitive, as it makes explicit the connection of such structure with the formulation of physical laws and, eventually, the notion of experience. There are two key questions for this type of project. First, which structures do represent spacetime in each theory. Second, how do they earn their spatiotemporal character. Although the two are related, my concern here is mainly with the second one: the question about how the spacetime structures in the different theories are generally justified. Simplifying, one might say that there are two potential general sources for justifying spacetime structures: either they are argued to be somehow introduced a priori in the formulation of the theory (this initially just meaning that they are taken to be determined previously to the formulation of the theory in question) or they are somehow to be justified by means of the laws of the theory. The two extremes are problematic: one seems to be forced either to deny the same demand of justification - admitting external elements - or to hide certain presuppositions needed for the formulation of the laws. ${ }^{13}$ Nonetheless, this might be a false dilemma: the a priori elements might be vindi-

${ }^{10}$ I leave this for a different paper.

11 The term trascendental, in this sense, has its origins in Kant. Nevertheless, given the variety of Kant's interpretations, the precise relationship between Kant's trascendental idealism and the present proposal is not a simple one. And this is not the place to discuss it.

12 Related approaches have a long tradition and more recent formulations like (DiSalle, 2006; Friedman, 2001).

${ }^{13}$ In a loose sense, the two extremes can be recognised in the two positions presented in the central dispute of this paper. 
cated as necessary for the formulation of laws that can give account of a certain field of experience and this, at the same time, would show the presuppositions hidden in the formulation of the laws for different theories. This is at the core of the strategy presented here as a middle path between the two extremes.

\subsection{SPACETIME Symmetries AND Symmetries OF LAWS}

Let us see how this general strategy can be of any help for the question about the explanatory relationship (if any) between spacetime symmetries and symmetries of laws. Recall that I argued before that the question about explanation, in absolute terms (bracketing issues about contextuality) cannot be answered without introducing ontology into the picture. My claim now is that this strategy allows a fruitful engagement with ontology in the context of the present discussion.

As we saw, the main challenge for a realist geometrical approach is to explain why matter laws must mimic the symmetries of spacetime structure, understood by the realist as an autonomous entity. Janssen's geometrical approach avoids this problem but with the result, as it was argued, of not being able to defend a position in which the arrow of explanation goes from spacetime to symmetries of laws. According to our perspective, the problem for the realist approach comes from abstracting spacetime structure from the set of physical events and then looking for an explanatory story that would go from this abstracted structure to the symmetries of laws. But one can take the origin of spacetime structure - with its symmetries - to be no other than the set of possible physical events.

The dynamical approach, on the other hand, makes explicit the necessity to provide an account for the requirement of symmetries of laws. For the strong constructivist version of this approach, on top of this, there is a demand to derive the full spacetime from non spatiotemporal assumptions. In any case, the pressing challenge is to justify that all the matter laws have the same symmetries without using an underlying spacetime structure as the last resource..$^{14}$ And this is precisely what is offered in the present approach, by putting the focus on the constraints on the formulation of physical laws and on the origins of such constraints.

The main idea of this general interpretive perspective, then, is the following: different physical theories introduce different restrictions on the formulation of physical laws by providing, implicitly, different definitions or characterisations of what a physical event is. Together with this, such definitions are linked (or they can be so a posteriori) to certain ways of justifying such constraints; ways that are related to general epistemic notions, certain conceptions of what physical experience is. The claim is that the introduction of spacetime notions have to do with this. Let us try to look at it in some detail.

Some restrictions are general for spacetime theories and have to do with basic features of physical experience or, in other words, with the basic conditions entering the definition of physical event. Formally these general restrictions are usually encoded in the topologi-

${ }^{14}$ As mentioned above, recent presentations of this approach avoid this challenge by explicitly claiming that for the dynamical approach this is an unexplainable brute fact in SR (the so-called first miracle of relativity) and arguing that no further explanation is forthcoming. See (Read et al., 2018) for an extensive defense of this position and (Sus, preprint) for a critical take on it. 
cal structure. They come from general assumptions about the content of physical experience: assumptions that obviously could be dropped at some point but that are operative for a number of theories. Take as an example relations of anteriority/posteriority and contiguity: we might say that it is part of the identity of a physical event that it is always next to, before or after other events. And this is something that is part of the type of experience that we have and that we attempt to capture with our physical theories.

The point coincidence argument is an example of an argument that attempts to justify certain general restrictions for theories. It starts by assuming that all physical theories can only describe coincidences of physical occurrences (ideally coincidences between system and measuring apparatus) to conclude that theories should be generally covariant. There were historically, and still at present, different readings of such an argument ${ }^{15}$ but a plausible minimal interpretation, that is actually realised in practice, is to see the argument as establishing that a topological manifold is a common structure of any admissible spacetime theory. ${ }^{16} \mathrm{We}$ see here how starting from some feature supposedly intrinsic to our notion of physical experience, some minimal condition for something to be empirically valid, we may say, a conclusion about the structure of the space of physical events described by physical laws is extracted. This illustrates the general idea: different conditions on the set of possible events that are introduced in the theories (some of them concomitant to some general conception of experience) and that can be read off the formalism conform the spatiotemporal character of the theory. So it is true, in a sense, that there are spatiotemporal assumptions that are put in the theories, as Norton argues against the strong constructivist program (we see this clearly in the case of the ones related to the topology of the space of events), but they must be understood as imposed with the scope of describing and delimiting certain type of experience. This does not show that the manifold is an autonomous entity on which metric spacetime must be built, it rather suggests that the formal conditions, implicit in a theory that tries to encode certain type of experience, must incorporate something like a topological structure that plays the role of a mechanism to individuate physical events.

But we have not said anything about the symmetries of laws yet. Physical events can be connected in different ways and different theories allow for different patterns of connectivity. This determines whether a given event can affect or be affected by any other. It seems desirable that this is well defined in a theory and, if it is, it provides what is often called its causal structure. In relativity, as we all know, this is given by the light cone structure derived from having a limit on the possibility of connecting two events by signals, and the empirical fact that light speed is independent from the speed of the emitter. So we naturally find that some principle about the connectivity of possible physical events generates certain structure that is part of what we call spacetime structure. Let us see how.

In the case of SR this stems from the two principles on which the theory was originally funded and from which the relativistic effects can be derived: the light principle and the relativity principle. The light principle, empirically, is based on the independence of the velocity of light from velocity of the emitter. Furthermore, on the theoretical side, Maxwell's

15 See (Giovanelli, 2013) for an excellent study of its different versions.

16 This does not mean that no theory can be formulated without a topological manifold, but that if it intends to make contact with experience, at some level of description, it will be describable with this topological space at its base. Perhaps this might be called, borrowing Huggett's terminology, phenomenal space. 
theory has solutions that can be interpreted as waves transmitted with constant velocity. This is the base that provides empirical content for a principle determining the connectivity between events and that will be valid for a certain class of observers. This latter fact, the restriction to a class of observers, is what the relativity principle takes care of, which rests, empirically, on Maxwell's equations being invariant under Lorentz transformations, Again this is the empirical fact that is elevated as principle in the form of the relativity principle. My claim, then, is that the origin of the requirement of the local Poincaré invariance of matter laws that is present in SR is to be found in this interpretation of the principles, as it imposes a restriction on the connectivity of events described by the different matter laws. The fact that light signals are used to determine the spatiotemporal relations between events restricts the local symmetries of laws (which describe relations between events) and conjunctly the local spatiotemporal symmetries.

Let me try to be more explicit about this last point. The two principles, based on the generalisaion of some features of light, impose the determination of the connectivity between events to be equally valid for a privileged class of observers; then this provides certain transformations that preserve such a structure: the spacetime symmetries. We need to see that such transformations are also the symmetries of the laws. The mentioned structure is a structure on the space of configurations of events allowed by the theory; this together with the types of fields and particles that enter the equations of the theory determines a space of kinematical possible models (KPM). Different matter theories, with their field equations and equations of motion, will select different models as solutions (different spaces of dynamical possible models), but all of them will have the structure of the space of KPM in common. And this determines the invariance properties of the equations of such theories in the following sense. We define the invariance group of a theory as the subgroup of the diffeomorphism group such that applied to the matter fields and particles of a solution of the equations generates another solution. ${ }^{17}$ This ensures that those transformations that leave the induced structure of the KPM invariant belong to the invariance group of the theory. Therefore, all the theories that can be formulated on the same space of KPM have the same subgroup of the group of diffeomorphisms as the invariance group, and it coincides with the group of transformations that are symmetries of the structure of the space of KPM. This shows, then, how symmetries of the matter laws are, at the same time, the symmetries of the emergent spacetime.

I must be clear about what I am not implying by this. I am not saying that any conceivable matter law must comply with these symmetries - which is trivially false- but that SR, as a theory, involves some principles — with an empirical base- from which the (local) symmetries of all matter laws and its coinciedence with spacetime symmetries can be derived. This means that, insofar as these principles are valid, and therefore the theory is adequate, the claims about symmetries of matter laws will hold. Note that SR, in fact, would claim such principles to be valid globally, but we now know that their validity, together with the symmetry claims, must be localised. The reverse of these claims is to say that SR, as any theory, through these principles defines a regime in which the theory is (approxiamately) correct.

Furthermore, we know that the structure on KPMs, introduced so far, does not determine Minkowski spacetime and that the symmetry associated with it is bigger than the

${ }^{17}$ See (Pooley, 2017; Sus, 2019) for more details on this. 
Poincaré group usually associated with the theory. The reason is that we have only considered the structure induced by the limiting connectivity between events (causal structure) and have not said anything about scale. To fix this, one should take into account that most physical theories address domains where scale is important, therefore they introduce this as part of the conditions on the definition of physical event. If this is the case, the fixed structure on the space of KPMs is the full Minkowski metric and the invariance group is the Poincaré group. This way we find certain symmetries which must be symmetries of any laws describing the connection between events precisely because they are symmetries of the structure recognised as spacetime.

As mentioned above, the program sketched here can be seen as the backbone of different proposals that, from long time ago, attempt at deriving relativistic spacetime structure starting from some principles related to causality. ${ }^{18}$ What they have in common is that they relate relativistic spacetime structure to some principles that have to do with general features about the connectivity of events (and the behaviour of certain bodies), although perhaps they do not make explicit the epistemological connection. I am not trying to defend that such principles are well justified, I am simply stating the fact that symmetries of laws and spacetime, in such approaches, can be seen as linked to this type of principles. ${ }^{19}$ Thus, whether one thinks that such principles are well founded or not, here we have a perspective that points to a common origin of spacetime symmetries and symmetries of laws.

\section{Responding to Norton's challenges}

Let us recall Norton's criticism to the strong constructivist program, criticism that he made extensive to any viable alternative to the naïve (my qualification) realist conception of Minkowski spacetime: it is not possible to derive spacetime structure starting from matter laws without introducing spatiotemporal assumptions, which would almost amount to presupposing the spacetime that allegedly was being derived. As I mentioned above, I think that Norton rightly points to some elements that seem necessary for the formulation of any matter theory from which one would hope to derive spacetime, and that such elements are somehow spatiotemporal. My disagreement has to do with how to judge this situation: I do not think, as he does, that the only way of justifying these presuppositions involves almost assuming the full spacetime structure and, therefore, I do not share his view of this as clearly supporting a naïve realist or substantivalist position.

Norton's first specific challenge has to do with the identity of events. I will formulate it in a way close to Norton's own words. Imagine that one starts from Lorentz invariance as a restriction on the formulation of any matter law and one wants to derive Minkowski spacetime from it. This presumes that the parameters that identify the different events introduced for any matter law are all the same. Then Norton asks: if one is not assuming that such parameters identify spacetime points, What is the base for their equality? Nothing, it seems. He supports his concern by noting that there are theories with other parameters,

18 Examples can be found in (Ehlers et al., 2012; Kauffman, 1985; Knuth et al., 2014; Lévy-Leblond, 1972; van Fraassen, 1970).

19 In (Sus, 2019) the constitutive character of such principles is thoroughly discussed. 
corresponding to internal degrees of freedom, for which the spatiotemporal character is not demanded.

As Norton recognises, his first concern is at least alleviated if one supposes a topological manifold that can be coordinatised to $\mathbf{R}^{4}$; and Pooley, as a defender of the dynamical approach, admits that this was always tacitly assumed in a potential construction of Minkowski spacetime. ${ }^{20}$ What is of interest for us, because this is what proves the efficiency of the present approach in solving the shortcomings of the dynamical approach, is to check whether there is a satisfactory story that justifies this common assumption present in almost every discussion of spacetime theories, or at least to see where we should look to provide one such a justification.

As it was stressed in the previous section, the leading idea here is that such justification has to do with general conditions on the notion of physical experience or, if one conceptualises this as being composed by physical events, on the definition of event. In the present case, the general characteristic has to do with what we might call unity of experience. If our theory admits different matter fields or particles, it is usually assumed that all of them can, in principle, interact with each other (whether they in fact do it or not); and this requires that there is a common pool of parameters in which such theories can be formulated. Of course, this restriction could be dropped, and then, as Norton suggests, different matter fields would define (and live in) different spaces and, at the same time, this would preclude that such different matter fields could influence each other. So it is not compulsory to assume this unity for the formulation of matter theories, but this is the case under a certain conception of physical experience that is operative for many, if not all, present physical theories. Furthermore, this perspective helps us with the question about the difference between internal and external spaces. It is true that some theories, even if introducing a common manifold of events, allow for internal spaces. The spacetime parameters have to do only with the possibility of mutual interaction, but not with the content or character of such interaction: to qualify these interactions, more internal degrees of freedom, encoded in these internal spaces, can be necessary.

It seems clear that, with the assumption of a unique underlying manifold of events, together with the mentioned assumption of unity of experience, come others like continuity, dimensionality, etc. My intention here is not to find plausible justification for them, but to state that they are correlated to more or less generalised and tacit assumptions about the nature of physical experience and that they are essential constituents of the definition of physical event operative in different physical theories. Take as an example the difference between spatial and temporal dimensions. It is ingrained in our conception of experience that things can happen at the same place, that something can change without moving or that it can move without changing essentially; well, any notion of event that can give account of such features seems to require at least a temporal and a spatial dimension. But this should not be confused with stating that we will never find a theory that does not presuppose this kind of structure: some time ago something like this could have been argued in favour of having an absolute notion of simultaneity or space being three dimensional, and today, in the light of some of our theories, these last features do not present themselves as necessary any more. The claim is not about necessity, aprioricity of psychological features

${ }^{20}$ Pooley's own reply to Norton's challenges can be found in (Pooley, 2013). 
of experience or anything of the kind; it is simply about such features being conditions defining the range of experience dealt with by certain theories and, at the same time, providing the justification for the emergence of certain space and time or spacetime in different theories. Some features are more basic, more ingrained and, therefore, shared by most of the theories, while others are more superficial and even linked to availability of certain measuring apparatuses.

The second of Norton's challenges for the constructivist program consists on pointing out that seeing spacetime as deriving from matter laws seems to be committed to a strong operational position. The idea is that for regions in which there is no matter content there seems to be nothing to which attribute the spatiotemporal properties. Norton provides what I think is the right scheme to answer this kind of worry: such assignments of spatiotemporal intervals to empty regions provide counterfactual statements about what would be measured if a material clock would connect the events. His criticism to this type of response seems to consist on stating that they do not match with his realist intuitions.

It is true, as Norton argues, that if one wants to derive spacetime from matter laws, one realises that some spatiotemporal assumptions enter, but also, I claim, that this does not mean that one is assuming the full spacetime that one is trying to derive. On the one hand, one recognises that the full metrical structure can be seen as, somehow, a consequence of some properties of matter laws, as the dynamical approach defends. On the other hand, there is a possible interpretation of the mentioned spatiotemporal assumptions that escapes from it being trivially question begging: first, the assumptions are conditions for the formulation of physical laws, second, such conditions are justified insofar as they are requirements that delimit some notion of physical experience.

\section{Conclusions}

I claim that the perspective presented in this paper supposes an important improvement with respect to both the geometrical and the dynamical approaches for the interpretation of $S R$ as it provides a better ontological setting for understanding the question about the nature of explanations of effects in spacetime theories.

First, let us look at the question about explanatory priority. As I argued in the first part of the paper, if the question is posed in terms of the arrow of explanation between spacetime symmetries and symmetries of laws and, moreover, one intends to be ontologically neutral, then there are no reasons to think that we should see symmetries of laws as explained by spacetime. What everybody seems to agree with, in the context of SR, is that the explanatory weight of typical relativistic effects falls on certain formal properties, but there is no reason for understanding such properties as being of a substratum different from whatever is described by the matter laws themselves. The main argument in defense of the explanatory priority of spacetime relies on the common origin inference and seems to presuppose a notion of formal explanation that, by itself, does not support the claim of the arrow going from spacetime to symmetries of laws. When the question is whether relativistic effects are explained either by spacetime structure or by Lorentz invariance, one can recognise that there are contexts in which saying that certain effects happen because spacetime is Minkowskian might be explanatory. But surely, the defenders of a non-dynamical approach want something stronger than that when they talk about the arrow of explanation 
between spacetime symmetries and symmetries of laws. And we are back to the starting point. On the other hand, there is an important pressure for the dynamical approach: if the question is about explanation, it seems ad hoc to take the symmetries of laws as brute facts.

There are two more or less direct morals that one might extract from this situation: first, leaving ontology aside does not seem to clarify anything and, second, the problem points to the question of how to explain that all matter laws should be Poincaré invariant. I have argued that these two issues -inserting ontology in the discussion and explaining what (Read et al., 2018) call the first of the miracles of relativity - are interdependent and that there is a strategy, with a long history but usually not explicitly exposed, that can show how to deal with them.

According to this strategy, the transcendental approach, symmetries of laws and symmetries of spacetime they both come from a common set of presuppositions implied in a theory: presuppositions that have to do with certain general requirements for physical experience and that are expressed by some general principles and materialised in the definition of physical events.

This also provides an explanation of why, in the relativistic context, all matter laws have the same local symmetries. The dynamical approach posses a challenge for the interpretation of SR: the explanation of relativistic effects by means of Minkowski spacetime is incomplete, so one needs to provide a more sophisticated account of the common symmetries of matter laws from which the effects can be derived. Janssen uses the COI to argue that Minkowski spacetime does actually explain the symmetries of laws, without being committed to a substantivalist understanding of spacetime. The transcendental approach presented here addresses this challenge differently, by providing a story of the origin of this common feature of laws; in a sense the search of common origin that Janssen asked for. But the answer, in this context, is not the Minkowski metric; which is also a product of the common origin. The answer comes from realising that every physical theory incorporates some general notion of experience that is materialised as a set of conditions on the formulations of the laws of the theory. This, in modern presentations of spacetime theories, can be seen captured as requirements ingrained in the characterisation of physical events, as conditions that are manifested in different layers of geometrical structures. For this strategy, the fact that all laws have the same symmetries, which such symmetries are and the account of the emergence of full spacetime have the same common origin. To complete the program one must be able to provide specific principles delimiting the notion of experience and see how they impose both symmetry restrictions on the formulation of laws and spacetime structure.

We have seen how some of these principles operate in SR and how they relate to the symmetries of spacetime and laws. There are some general conditions on the same definition of event, supported by some principles on physical experience. This endows the set of events with topological properties; the claim is that such properties are justified insofar as certain conception of what is admissible physical experience is operative; which, no doubt, is dependent on what the world is like. I have mentioned explicitly what I have called principle of unity of experience because it allowed me to reply to some concerns about the plausibility of the constructive program, but other principles could be presented in this context. What is important here is to realize how general formal conditions, usually accepted for the formulation of spacetime theories, have these kind of principles behind. The ones encoded by the topological manifold of events are shared 
by many theories, not just SR, which explains why the standard formulation of spacetime theories is so spread. But note that under this perspective, the mentioned principles are not just seen as equivalent to the mere acceptance of a given spacetime structure of the naïe realist, neither as arbitrary phenomenological principles, they are conditions for the formulations of physical laws. ${ }^{21}$ Furthermore, we also have some principles that restrict the possible connections of different events: in the case of SR, we can bring to the fore something like a limiting signaling principle (empirically sustained by the constancy of the velocity of light). This is the base from which the symmetries of matter laws (encoding relations between events) can be derived together with the metrical structure of Minkowski spacetime (as the one surveyed by measuring devices behaving in accord with Poincaré invariant laws).

The present strategy provides, then, a peculiar response to the problem of the relation between the dynamical symmetries and the spacetime symmetries and a middle path between the naive realist and constructivist positions. I think that it shares the spirit of the dynamical approach: spacetime is given as a set of general conditions for the formulation of physical laws. And it is genuinely realist about spacetime, but without the need to postulate any magical explanatory relation between it and phenomena.

What kind of account of spacetime does this perspective endorse? Spacetime is presented as somehow derived from certain general conditions on the formulation of laws, which can be viewed as part of the definition of event implicated in a given notion of physical experience. Does this mean that, under this view, spacetime is explained? If it is, explained by what? And, according to what notion of explanation? As I see it, this perspective provides a framework to understand the relation between spacetime structure and symmetries of physical laws in different theories. In this sense, it provides the ontological scaffolding that supports the claims of explanations going from spacetime to some effects through symmetries of laws, in some contexts, and also the resistance, sometimes found, to accept that spacetime is part of the explanation. All this is done, as I have stressed, by seeing spacetime as in a way constructed from events that fit to certain notion of experience that is encoded in physical laws.

\section{Acknowledgements}

I would like to thank some anonymous reviewers for their comments. Research for this article has been supported by the following projects: "Laws, explanation and realism in physical and biomedical sciences" (FFI2016-76799-P) and "Limits of quantum physics - formalism, interpretation, visualization and aesthetics” (FFI2016-77266-P), Ministerio de Economía y Competitividad (Spain).

21 The discussion of the status of these principles is fundamental for this type of approach. Examples of such a discussion can be found in (DiSalle, 2006; Friedman, 2001; Sus, 2019). Further work is needed in this area. 


\section{REFERENCES}

Acuña, P. (2016). Minkowski spacetime and Lorentz invariance: The cart and the horse or two sides of a single coin? Studies in History and Philosophy of Modern Physics 55, 1-12.

Balashov, I., Janssen, M. (2003). Presentism and relativity. British Journal for the Philosophy of Science 54, 327-346.

Bokulich, Alisa (2011). How scientific models can explain. Synthese, 180 (1):33-45.

Brown, H.R. (2005). Physical Relativity: space-time structure from a dynamical perspective. Oxford: Oxford University Press.

Brown, H.R., Pooley, O. (2006). Minkowski space-time: A glorious non-entity. In: D. Dieks (ed.) The ontology of spacetime (pp. 67-89). Amsterdam: Elsevier.

Brown, H., Read, J. (Forthcoming). The dynamical approach to spacetime theories. In E. Knox and A. Wilson (eds.), The Routledge Companion to Philosophy of Physics. London: Routledge.

Einstein, A. (1905). Zur elektrodynamik bewegter Körper. Annalen der Physik 17, 891-921.

Einstein, A. (1919). What is the theory of relativity? London Times, 28 November, Repr. in CPAE, Vol. 7 , Doc. 25.

Disalle, R. (2006). Understanding spacetime: The philosophical development of physics from Newton to Einstein. Cambridge: Cambridge University Press.

Dorato, M. (2015). Events and the Ontology of Quantum Mechanics. Topoi 34, 2, 369-378.

Dorato, M., Felline, L. (2010). Structural explanations in Minkowski spacetime: which account of models? In: V. Petkov (ed.) Space, Time, and Spacetime: Physical and Philosophical Implications of Minkowski's Unification of Space and Time (pp. 181-192). Amsterdam: Springer.

Ehlers, J., Pirani, F.A.E., Schild, A. (2012). Gen Relativ Gravit 44: 1587. doi:10.1007/s10714-012-1353-4.

Felline, L. (2011). Scientific explanation between principle and constructive theories. Philosophy of Science 78, 989-1000.

Felline, L. (2015). Mechanisms meet structural explanation. Synthese 195(1), 99-114. doi: 10.1007/s11229015-0746-9.

Friedman, M. (2001). Dynamics of Reason. Stanford: CSLI Publications.

Frisch, M. (2011). Principle or constructive relativity. Studies in History and Philosophy of Modern Physics 42, 176-183.

Giovanelli, M. (2013). Erich Kretschmann as a proto-logical-empiricist: Adventures and misadventures of the point-coincidence argument. Studies in History and Philosophy of Modern Physics 44, 115-134.

Janssen, M. (2002). COI stories: Explanation and evidence in the history of science. Perspectives on Science, $10,457-522$.

Janssen, M. (2009). Drawing the line between kinematics and dynamics in special relativity. Studies in History and Philosophy of Modern Physics 40, 25-52.

Kauffman, L.H. (1985). Transformations in Special Relativity. International Journal of Theoretical Physics, 24, 3.

Knuth, K. H., Bahreyni, N. (2014). A potential foundation for emergent space-time. Journal of Mathematical Physics 55(11), 112501, doi: 10.1063/1.4899081.

Lévy-Leblond, J.M. (1976). One more drivation of the Lorentz transformation. American Journal of Physics 44, 271, doi: 10.1119/1.10490.

Myrvold, W. C. (2019). How could relativity be anything other than physical? Studies in History and Philosophy of Modern Physics 67, 137-143. http://dx.doi.org/10.1016/j.shpsb.2017.05.007

Norton, J. (2008). Why constructive relativity fails. British Journal for the Philosophy of Science 59, 821-834.

Pooley, O. (2013). Substantivalist and Relationalist Approaches to Spacetime. In: Robert Batterman (ed.). The Oxford Handbook of Philosophy of Physics. Oxford: Oxford University Press.

Pooley, O. (2017). Background Independence, Diffeomorphism Invariance, and the Meaning of Coordinates. In: D. Lehmkuhl, G. Schiemann, E. Scholz (eds.). Towards a Theory of Spacetime Theories. Berlin: Springer. 
Read, J. (2019). On miracles and spacetime. Studies in History and Philosophy of Modern Physics 65, 103-111. https://doi.org/10.1016/j.shpsb.2018.10.002

Read, J. (Preprint). Explanation, geometry and conspiracy in relativity theory.

Read, J., Brown, H. Lehmkuhl, D. (2018). Two miracles of general relativity. Studies in History and Philosophy of Modern Physics 64, 14-25. https://doi.org/10.1016/j.shpsb.2018.03.001

Sus, A. (2019). Explanation, analyticity and constitutive principles in spacetime theories. Studies in History and Philosophy of Modern Physics 65, 15-24. https://doi.org/10.1016/j.shpsb.2018.08.002

Sus, A. (Preprint). Relativity without miracles.

Van Fraassen, B. (1970). An Introduction to the Philosophy of Time and Space. New York: Random House.

Adán Sus is a philosopher of physics at the Department of Philosophy of the University of Valladolid. His research has focused mainly on the philosophy of spacetime theories. He has published on questions related to the interpretation of relativity theory, the status of its principles and the role of symmetries in physics.

Address: Departamento de Filosofía, Universidad de Valladolid. Plaza del Campus, s/n (47011 Valladolid-España). E-mail: adansus@fyl.uva.es. ORCID: 0000-0002-2097-1192. 